\title{
¿CUÁLES SON LOS ÁMBITOS DE TRANSVERSALIDAD EDUCATIVA?
}

\section{WHAT ARE THE AREAS OF EDUCATIONAL TRANSVERSALITY?}

\author{
http://dx.doi.org/10.15304/ie.29.6023
}

\author{
Carlos Rosales López \\ Universidade de Santiago de Compostela \\ carlos.rosales@usc.es
}

\section{RESUMEN}

En este trabajo se analizan las características de la transversalidad educativa en una serie de ámbitos como la organización de contenidos de enseñanza, la planificación administrativa, el trabajo en los centros escolares y en las aulas, los recursos o medios educativos y los modelos de innovación.

Se hace uso de una metodología basada en la reflexión sobre la realidad educativa actual en los niveles de educación infantil, primaria y secundaria y en el análisis de obras relevantes en el campo, así como de documentos y programas de carácter internacional y la legislación en un sentido histórico y administrativo, con estudio específico del tratamiento de los temas transversales en las leyes orgánicas correspondientes a las últimas décadas en nuestro país: LODE (1985), LOGSE (1990), LOE (2006) y LOMCE (2013).

En la organización de contenidos se pone de relieve la necesidad de potenciar el trabajo interdisciplinar y en equipo, capaz de superar los límites de la organización disciplinar convencional. Respecto a la planificación administrativa, se subraya su carácter ambiguo y poco orientador, con mayor incidencia a nivel de fines, principios y objetivos, pero no tanto de contenidos, con notables desequilibrios en el tratamiento de diversos temas.

Se considera que el centro escolar puede plasmar en su proyecto educativo una planificación adaptada de los temas transversales. Y que el profesor, como profesional capacitado para conducir los procesos de enseñanza y aprendizaje, puede hacer realidad el desarrollo de los mismos en las tareas cotidianas del aula. Se propone que la innovación educativa en la enseñanza de los temas transversales combine el modelo descendente, promovido por la administración educativa, con el ascendente, constituido por iniciativas valiosas de profesores y centros y el horizontal, cuando instituciones y centros se coordinan y cooperan en proyectos que parten de necesidades comunes y asumen aspiraciones semejantes.

Palabras clave: Temas transversales, planificación, función docente, recursos, innovación.

\begin{abstract}
This paper analyzes the characteristics of educational transversality in a series of areas such as the organization of teaching content, administrative planning, work in schools and classrooms, resources or educational media and innovation models.
\end{abstract}

Recibido: 09/V/2019. Aceptado: XI/2019 
It is used a methodology based on the reflection on the current educational reality at the levels of preschool, primary and secondary education and on the analysis of relevant works in the field, as well as documents and programs of an international nature and legislation in an historical and administrative sense, with specific study of the treatment of transversal themes in the organic laws corresponding to the last decades in our country: LODE (1985), LOGSE (1990), LOE (2006) and LOMCE (2013).

The organization of contents highlights the need to promote interdisciplinary work and in teams, capable of overcoming the limits of conventional disciplinary organization. With regard to administrative planning, its ambiguous and unguiding nature is underlined, with greater incidence at the level of goals, principles and objectives, but not so much content, with notable imbalances in the treatment of various issues.

It is considered that the school can translate into its educational project an adapted planning of transversal themes. And that the teacher, as a trained professional to redirect the teaching and learning processes, can make the development of them in the daily tasks of the classroom a reality.

It is proposed that educational innovation in the teaching of transversal themes combine the descending model, promoted by the educational administration, with the ascendant, constituted by valuable initiatives of teachers and centers and the horizontal, when institutions and centers coordinate and cooperate in projects that start from common needs and assume similar aspirations.

Keywords: Transversal themes, planning, teaching function, resources, innovation.

\section{ORGANIZACIÓN DE CONTENIDOS}

En las sociedades menos evolucionadas técnicamente, la transmisión del saber a las nuevas generaciones se realiza mediante la participación directa en las experiencias vitales y a través de la comunicación oral. Se puede considerar que en ellas es toda la comunidad la que asume una responsabilidad educativa y se estima asimismo que la desaparición de las personas mayores, que han acumulado gran cantidad de conocimientos y experiencia, constituye una importante pérdida para la cultura y su transmisión.

La invención y desarrollo de la escritura permitió la acumulación de ingentes cantidades de información de los más diversos ámbitos del conocimiento y su protección frente al riesgo de pérdida por olvido. (Cavallo y Chartier, 2001; Clemente, 2004). Con el paso del tiempo aparecen las bibliotecas y las escuelas, como instituciones depositarias del conocimiento y responsabilizadas de su conservación, difusión y transmisión. El conocimiento se estructura y clasifica en diferentes ámbitos. Se realizan tareas de organización racional, de actualización, de traducción...En las escuelas, los profesores clasifican los contenidos a enseñar siguiendo de forma predominante la misma estructura lógica del conocimiento científico y técnico que utilizaban los eruditos e investigadores. Así, a las materias clásicas de lengua (lectura y escritura) y matemáticas (cálculo), se van agregando las ciencias naturales y sociales, la educación artística, la educación física, etc.

La separación de campos de conocimiento y aprendizaje se hace cada vez mayor, especialmente cuando las escuelas unitarias, en las que un solo profesor impartía todos o la mayor parte de contenidos, son sustituidas por las escuelas graduadas o grandes centros escolares con diferentes materias. Se incrementa la separación entre ámbitos en favor de la especialización docente. Ocurre con frecuencia que la enseñanza se configura como un conjunto de compartimentos estancos, cuya separación se hace patente en horarios rígidos de trabajo. Los profesores hacen valer su propio campo, estableciéndose así como una especie de competición entre unos y otros. 
Las consecuencias de este vertiginoso proceso de diferenciación, son considerablemente negativas desde la perspectiva de la formación completa de niños y jóvenes. Se pierde en el horizonte escolar la necesidad de una educación a través de la participación activa en los procesos de elaboración del conocimiento. En las escuelas a los alumnos se les separa de la realidad y se les pone en contacto con el conocimiento ya elaborado, pero en cierto modo, desvitalizado. Se les ofrece una visión fragmentada y jerarquizada de la realidad. Se les evalúa por el conocimiento de solo determinadas dimensiones de la misma.

Manifestaciones de esta realidad se ponen de relieve en estos momentos, cuando pruebas externas en el sistema educativo a nivel nacional e internacional (LOMCE, 2013; PISA, 2018) se proyectan en exclusiva o de forma predominante en los ámbitos de la lengua, las matemáticas y las ciencias. ¿Se ha valorado el efecto pedagógico negativo que esta práctica puede tener en el desarrollo de contenidos vinculados con la educación física, la expresión artística, la convivencia, la salud, etc.?

En la organización disciplinar convencional de los contenidos, se ha asociado la responsabilidad docente al ámbito de una o varias materias y se ha dado lugar al fortalecimiento del trabajo individual de los profesores. Con el paso del tiempo, sin embargo, se ha puesto de relieve la existencia de importantes contenidos de enseñanza y aprendizaje no contemplados en el marco de las disciplinas convencionales. Se trata de relevantes cuestiones de carácter social y personal que como la convivencia, el medio ambiente, la salud, la igualdad hombre-mujer y otros, contienen un valor pedagógico desbordante, difícilmente abarcable en el ámbito de una sola materia o de un conjunto limitado de temas.

La escuela al tiempo, como institución creada en el seno de una comunidad social, presenta en si misma unas características que en gran medida constituyen un reflejo de la sociedad. Esta en las últimas décadas evoluciona hacia un progresivo reconocimiento de los derechos humanos y las relaciones democráticas, con aspiración a la inclusión. Y en este sentido, también la escuela ha de resolver constructivamente el proceso de inclusión de alumnos que presentan diversas características étnicas y culturales. Se constituye en efecto, en un escenario de vida en el que niños y jóvenes van a interactuar durante muchas horas, días y años, a través de la realización de actividades de carácter formal e informal. (Rosales, 2011; Pujolás, 2008).

La tarea de los centros escolares se complica pues, de tal manera que el esquema convencional de organización disciplinar y responsabilidades individuales, se rompe en función del desarrollo de nuevas formas de trabajo interdisciplinar y colaborativo. $Y$ es precisamente en este punto en el que surge con fuerza el concepto de temas transversales, como contenidos que "atraviesan" las distintas materias convencionales, que las transcienden y las llegan a modificar sustancialmente (Rosales, 2015).

\section{PLANIFICACIÓN CONVENCIONAL}

Las administraciones educativas, de acuerdo con un principio de racionalidad técnica y en función de lograr resultados cada vez más constatables y eficaces en la actuación de los sistemas educativos, de los centros escolares y los profesores, vienen promoviendo de manera sistemática 
desde mediados del siglo XX un modelo de planificación de la enseñanza cuyos componentes básicos son principios y fines, objetivos, competencias, contenidos y orientaciones metodológicas y de evaluación.

Existen diversos niveles de planificación e intervención. Así, en principio, el representado por organizaciones internacionales como la ONU, la UNESCO o el Consejo de Europa. Otro nivel se corresponde con las administraciones estatales o nacionales, que se manifiesta a través de leyes educativas fundamentales como en España la LODE (1985), la LOGSE (1990), la LOE (2006) y la LOMCE (2013). El siguiente nivel de concreción corresponde a las administraciones regionales o autonómicas. En este caso las disposiciones administrativas reflejan y adaptan al entorno sociocultural próximo las orientaciones contempladas en las leyes estatales, así como estas constituyen una proyección de las grandes orientaciones internacionales.

Dentro el ámbito de intervención de las administraciones regionales o autonómicas se desenvuelve la actividad de las administraciones locales y más concretamente de los centros escolares y los profesores, que cuentan como instrumento mediador con la administración autonómica con los centros de profesores y recursos.

La capacidad de toma de decisiones de cada uno de los niveles e instituciones citados es variable en función del predominio de estrategias más o menos centralizadas o descentralizadas en los sistemas educativos. Países claramente centralizados en Europa lo han sido durante mucho tiempo Francia y Austria, así como en el extremo de descentralización se han destacado otros como Bélgica y Dinamarca. España, que en la época de la dictadura estuvo rígidamente centralizada, en el periodo de transición a la democracia, ha descentralizado considerablemente la toma de decisiones educativas, confiriendo una progresiva capacidad de decisión a las diversas Comunidades Autónomas y en alguna medida también, a los centros escolares y a los profesores.

No obstante, es patente la continuidad de una línea racional técnica muy convencional, en la planificación de la intervención educativa en todos los niveles de la administración educativa. Así, en los fines, principios y objetivos generales de las leyes recientes, tanto en la LOGSE (1990), como en la LOE (2006) y en la LOMCE (2013), se hace hincapié en la formación completa de los alumnos. Ahora bien, en la organización de contenidos de enseñanza, se sigue un modelo convencional de diferenciación según materias o asignaturas clásicas. Esta organización convencional deja fuera del campo curricular, de los horarios lectivos, el tratamiento de importantes temas o contenidos de carácter social, fundamentales para el desarrollo completo de la persona, tal como se propone en fines, principios, objetivos y competencias.

La LOGSE (1990), tras realizar una organización convencional de los contenidos, proponía como solución a este problema, la introducción y desarrollo implícito en las materias convencionales, de temas como:

Educación moral y cívica, educación para la paz, educación para la igualdad hombre-mujer, educación sexual, educación para la salud, educación ambiental, educación para el consumo y educación vial.

En esta ley de hecho, se consideraba que el tratamiento de estas cuestiones transversales en todas las disciplinas, daría lugar a una renovación cualitativa de las mismas (una historia de paz y 
no de guerras, ciencias sensibles con el medio ambiente, lenguaje en el que se analizan textos sobre cuestiones de gran actualidad e interés social, etc.).

La LOE (2006) con posterioridad, reafirma esta línea de tratamiento implícito, pero introduce importantes novedades. En primer lugar, se implanta una asignatura (educación para la ciudadanía y los derechos humanos) en las etapas de educación primaria y secundaria obligatoria (ESO). Esta asignatura era obligatoria y por lo tanto, la deberían estudiar todos los alumnos. En ella se trabajarían importantes cuestiones vinculadas con la convivencia, la paz, la igualdad y los derechos humanos.

Por otra parte la LOE también incluye como temas transversales los lenguajes verbal, audiovisual e informático. Se trata de una importante novedad. Así como la LOGSE había considerado como temas transversales cuestiones de relevante interés social y personal, en el caso de la LOE, se incide también en temas o contenidos académicos clásicos y actuales (lenguajes), reconociendo que su enseñanza debe constituir una responsabilidad compartida, más allá de los márgenes de determinadas asignaturas convencionales.

En la LOMCE (2013), con el término de “elementos transversales” se hace referencia a los considerados así en las dos anteriores leyes orgánicas. Con la LOMCE desaparece la asignatura obligatoria de educación para la ciudadanía y se crea una optativa denominada educación en valores. Se modifica el peso o intensidad de algunos temas transversales anteriores y se incluyen otros nuevos. Quizás, la principal novedad aportada por la ley en este sentido, es la consideración de la educación inclusiva como tema transversal, es decir, como responsabilidad compartida por toda la comunidad educativa.

Ahora bien, así como es evidente la mención de los temas transversales en fines, principios, objetivos y competencias, sin embargo en el ámbito de los núcleos de contenido se podría decir que su presencia se diluye y solo puntualmente aparecen determinados temas. Así, en el Decreto de Currículo para Educación Primaria de la Xunta de Galicia (2014) se constata que:

- En ciencias sociales, con cuatro bloques de contenido, tan solo se consideran en el tercero: Aprender a vivir.

- En Ciencias naturales, con cinco bloques de contenido, tan solo aparecen en el segundo: El ser humano y la salud

- En lengua castellana y literatura, con cuatro bloques, en el primero se hace referencia a la comunicación oral: Aprendizaje integrado de las lenguas en sociedades plurilingües

- En matemáticas, con cinco bloques, no aparecen en ninguno.

- En primera lengua extranjera, con cinco bloques, en el quinto se trata: El conocimiento de la lengua y convivencia intercultural.

Es fácil comprender que esta inclusión puntual de los temas transversales da lugar a un tratamiento insuficiente de los mismos, no solo desde una perspectiva cuantitativa, sino en cuanto a posibles dimensiones a considerar en cada uno de ellos. Por ejemplo, en todo el citado Decreto se propone un tratamiento de la educación para la salud desde las perspectivas de la alimentación y el 
ejercicio físico, pero no se hace referencia a una salud mental y social y los temas de drogodependencia, educación sexual y educación para el consumo entre otros, prácticamente no se mencionan.

\section{LA INTERVENCIÓN DEL PROFESOR}

La planificación que un equipo docente realiza a medio o largo plazo sobre las tareas de enseñanza y aprendizaje, constituye una orientación a seguir por cada profesor en el contexto de su interacción directa con los alumnos. Ahora bien, el desarrollo cotidiano de las tareas en el aula le obligará a adoptar constantemente decisiones específicas, de carácter interactivo, que responderán fundamentalmente a las actitudes y comportamientos de sus alumnos (comunicación, comprensión, motivación...).

Las decisiones de intervención inmediata del profesor, también pueden surgir de su propia iniciativa, en el horizonte de una optimización de las tareas cotidianas o pueden venir motivadas por sucesos ocasionales no imaginables en una planificación previa, sucesos que sin embargo a veces ejercen una importante influencia sobre sus actividades y las de sus alumnos. La dinámica relacional en el aula, constituye un factor ambiental de notable influencia sobre las decisiones docentes. Cada grupo, cada clase presenta su propia personalidad social, que evoluciona con el tiempo y condiciona las decisiones y actuaciones de sus miembros.

De aquí que se haya utilizado el término de “enseñanza emergente" para designar las características del perfil que finalmente adquiere la enseñanza en la realidad cotidiana de las aulas. Grandes representantes del interés de la enseñanza emergente han sido J. Dewey (aprender haciendo), L. Stenhouse, (programación como establecimiento de principios de procedimiento $\mathrm{u}$ orientaciones generales) y J. Bruner (programación en espiral frente a yuxtapuesta). Así como en la planificación convencional los objetivos presentan un papel estelar, punto de partida para la caracterización de la actividad, desde la perspectiva de la enseñanza emergente se confiere también importancia a componentes del proceso didáctico como los contenidos, las actividades, los recursos y la evaluación, componentes que en la planificación convencional aparecen claramente subordinados a la naturaleza de los objetivos. Todo ello desde la consideración de la capacidad del profesor para reconstruir en cada momento la naturaleza de los procesos educativos. En relación con la planificación previa, se considera que las decisiones que conforman la enseñanza emergente pueden modificar o consolidar las orientaciones que en ella se habían previsto, pero que en todo caso las vienen a especificar y dar vida.

La enseñanza y aprendizaje de los temas transversales puede contemplarse en las planificaciones a medio y largo plazo, y ello constituye una garantía de su presencia en las aulas. Pero la calidad de dicha presencia y de su desarrollo, dependen en gran medida de la capacidad del profesor para incardinarlos en las tareas cotidianas. Y podríamos analizar esta capacidad docente desde diversas perspectivas como su trabajo en equipo con otros profesores y especialistas, su tarea clásica de transmisión de conocimiento, su dedicación como educador en valores y su función orientadora en relación con el grupo y con cada alumno (tutor). 
El trabajo en equipo constituye la gran ocasión para coordinarse con otros profesores y profesionales en la planificación, el seguimiento y la evaluación de programas y proyectos. En el caso de los temas transversales, dado su carácter multidisciplinar, esta tarea de coordinación es imprescindible si se quiere lograr una intervención coherente y no fragmentada, si se quiere dar el paso cualitativo tan necesario de la multidisciplinaridad a la interdisciplinaridad.

La función del profesor como enseñante, la transmisión de conocimiento en torno a los temas transversales, constituye una primera tarea fundamental, punto de partida para la estimulación de aprendizajes vinculados con las habilidades, las actitudes y las competencias. El nivel madurativo de sus alumnos, su nivel académico y sus centros de interés predominantes, deberán constituir criterios a considerar por el profesor en la selección de conocimientos. Para su trabajo en el aula puede seguir una línea sistemática vinculándolos a contenidos presentes en las distintas materias y áreas curriculares. Puede utilizar también unidades didácticas expresamente proyectadas sobre determinados temas o puede incardinarlos al trabajo sobre sucesos especialmente relevantes en su contexto social y natural.

Ahora bien, el profesor debe asumir una especial responsabilidad en la inducción en sus alumnos no solo de conocimientos, sino también de habilidades, valores y competencias. Y esto no parece posible sin la utilización de estrategias y técnicas que impliquen una fuerte dedicación personal y social tanto fuera como dentro del aula. En este sentido, parece necesario que manifieste una actitud positiva y un comportamiento coherente con los aprendizajes que trata de estimular en sus alumnos. No tendría sentido que, ante un conflicto entre alumnos, no intentara mediar y dialogar, sino que delegara la solución en otras instancias o recurriera a la aplicación formal de normas y sanciones existentes, contempladas para tales casos. No sería coherente impartir conocimientos sobre la igualdad, pero de hecho practicar la discriminación en su relación con el alumnado. No tendría sentido hablar a sus alumnos del interés de la ayuda mutua dentro de un sistema individualista y competitivo de trabajo. No tendría sentido reconocer la importancia de la paz en el mundo desarrollando una historia en la que tienen protagonismo las batallas, los caudillos...

Hacer compatible el aprendizaje de contenidos transversales (conocimientos y valores) con los contenidos convencionales dentro del mismo espacio curricular, implica de hecho llevar a cabo una actualización social y cultural de las materias convencionales, desarrollarlas con una proyección personal y social vivas. Posiblemente, solo los profesores con una profunda convicción y actitud positiva hacia los temas transversales, estarán en situación de llevar a cabo esta transformación. Por el contrario, muchos profesores seguirán priorizando los contenidos clásicos, pensando que si dedican tiempo a las nuevas cuestiones transversales, no van a poder desarrollar al completo el programa de su asignatura.

La función orientadora y tutorial del profesor presenta una doble proyección: sobre su grupo de alumnos y sobre cada uno de los mismos. Temas como la convivencia, el medio ambiente, la igualdad o el consumo, presentan una clara proyección grupal. Cada docente debería conocer cuál es la preparación general de su grupo de alumnos en estos temas y programar formas de intervención adaptadas. Al mismo tiempo, los temas transversales presentan unas características específicas en cada alumno, por lo que es necesaria además una intervención tutorial personalizada, en la que el docente debe contar con la información/colaboración de los padres. 
Es necesario advertir que esta función tutorial está estrechamente vinculada a su tarea planificadora y al desarrollo del plan de orientación y acción tutorial del centro educativo. En los primeros cursos de educación primaria, un profesor es responsable de la mayoría de contenidos curriculares y trabaja con el mismo grupo de alumnos la mayor parte del horario escolar.

En la realización de tareas de intervención docente y tutorial, en la reconducción del desarrollo adaptado de la enseñanza de los temas transversales, es precisa una constante puesta al día sobre las necesidades de aprendizaje de los alumnos y sobre sus procesos de aprendizaje. Se puede hacer referencia a diversos instrumentos de información, como la conversación y el debate en grupo, la entrevista semiestructurada y la observación en sus diferentes modalidades...

Desde una perspectiva etnográfica, presenta especial interés la práctica de la observación naturalista, es decir, aquella que el profesor, como miembro del grupo realiza de forma paralela al desarrollo de las actividades del mismo, sin interferir en ellas. Una observación que se puede realizar durante largos periodos de tiempo, que permite al profesor interpretar de forma contextualizada aquello que observa en el lugar y tiempo en que tiene lugar y que está abierta a recoger datos sobre todas las posibles formas de actuación, convencionales o no, previstas o imprevistas, que puedan presentar cierta significación pedagógica/educativa.

La observación naturalista, al abrirse a la captación de lo inesperado, constituye un valioso instrumento para la resolución educativa de problemas didácticos o incidentes críticos (Rosales, 2017), a partir de cuya descripción y análisis se puede estimular el aprendizaje sobre temas transversales en el aula.

\section{FORMACIÓN DOCENTE}

Si se considera que los temas transversales constituyen aprendizajes valiosos a desarrollar a nivel de educación primaria, en la formación del profesorado debería contemplarse su capacitación para la enseñanza de los mismos. (Ávila, 2016) Es más, ya en los procesos de selección para los estudios de profesorado es preciso considerar actividades y actitudes positivas hacia estos temas, como se ponen de relieve a través de actividades de voluntariado, de servicio de carácter social y humanitario. (Zeichner, 2010).

Una primera característica a considerar en la formación inicial del profesorado se refiere a la presencia, también transversal de estos temas en su plan de estudios, aunque no es descartable su introducción explícita en los mismos. De hecho, así ocurre en el Decreto de 2007 que regula las enseñanzas universitarias, en el que se dispone que deberán incluir: "Los derechos humanos, los principios democráticos, los principios de igualdad hombre-mujer, de solidaridad, de protección medioambiental, fomento de una cultura de paz".

En el Estatuto del Estudiante Universitario, de 2010, se considera que se deben incluir objetivos de formación personal y en valores. En las órdenes ministeriales sobre planes de estudio (Orden ECI 3857/2007) se incluyen asimismo competencias transversales.

Ahora bien, igual que ocurre en el currículo de educación primaria, esta presencia general de los temas en los planes de formación inicial del profesorado a nivel de objetivos y su consideración 
puntual en ciertos núcleos de contenido, no constituye garantía suficiente para un desarrollo completo de los mismos en la formación de futuros maestros/as. Es necesario un tratamiento también transversal que se manifieste en todos los contenidos y de manera especial, mediante una mayor aproximación de los estudios a la realidad sociocultural.

En cuanto a la formación permanente del profesorado, se podría pensar en la conveniencia de incrementar actividades de carácter convencional como cursos, seminarios, jornadas, a través por ejemplo, de los Centros de Profesores y Recursos. Habría que hacer referencia en este caso, a la optimización de las características de estas actividades a través de una adecuada programación según necesidades, una mayor participación del profesorado, una cuidadosa elección de los responsables de impartirlos, una evaluación diferida de los resultados.

Ahora bien, las condiciones de los centros escolares para desarrollar estos temas varían considerablemente. Cada centro presenta una personalidad propia derivadas de las características de sus alumnos, de la formación de sus profesores, de los recursos disponibles, de los vínculos que mantiene con la comunidad... Sin embargo, la formación y capacitación que cada profesor adquiere en el contexto de su currículo personal, no responde siempre a las expectativas del centro en el que trabaja.

Por todo ello, parece conveniente estimular y apoyar la realización de proyectos de perfeccionamiento en los propios centros educativos. Se favorece así una coordinación de trayectorias individuales con la mejora global del centro. Se incrementa la responsabilización de los profesionales y se favorece la aplicación directa de los aprendizajes en la propia realidad. Se podría citar por ejemplo, un centro con elevado número de alumnos de diversos orígenes étnicos y culturales que emprende un proyecto de formación para la inclusión. $\mathrm{O}$ el caso de un colegio con una grave problemática medioambiental, que emprende un proyecto de formación del profesorado para el tratamiento pedagógico de este tema en las aulas.

\section{DIVERSIDAD DE MEDIOS}

El plan lector de los centros y los temas transversales presentan una estrecha vinculación. La Administración educativa, en línea con orientaciones de ámbito internacional, se ha hecho especialmente consciente durante las últimas décadas, de la importante influencia que tiene la lectura comprensiva en la estimulación del aprendizaje y en la formación del alumno, en general. Por ello, en la LOE (2006), se dispone que todos los centros educativos elaboren un plan lector y que en todas las materias o áreas curriculares se dedique un tiempo a la lectura. En la estimulación de la capacidad de lectura comprensiva se han de ver implicados todos los profesores y en general, toda la comunidad educativa. La lectura se convierte así en un tema transversal y no solo con material impreso, sino también en sus versiones audiovisual y digital.

Un componente nuclear de los planes lectores son las bibliotecas de centro y de aula. En ellas deben existir medios impresos, audiovisuales y digitalizados para la lectura. Estos medios se van adquiriendo de manera paulatina por cada centro a través de la utilización de sus propios fondos o del intercambio. Y es necesario que por parte de la gestión de las bibliotecas exista un cierto interés por dotarlas de recursos sobre los temas transversales. 
Sugerencias de profesores y alumnos pueden constituir una fuente de orientaciones en dichas adquisiciones. En todo caso, es evidente que importantes temas sociales como la convivencia, el medio ambiente, la salud, la igualdad, la prevención de drogodependencias, la seguridad vial o el consumo, deberían estar convenientemente representados.

La biblioteca podría/debería convertirse en un centro de documentación sobre estos temas. En su gestión, en la medida en que se cuente con tiempo liberado de docencia, se podría solicitar información y medios a diversos organismos internacionales y nacionales/autonómicos, oficiales y no gubernamentales, que en estos momentos trabajan y elaboran valiosos recursos (informes, unidades didácticas, vídeos, guías docentes...) y que suelen poner a disposición de colegios y otras instituciones sociales. De notable interés son los recursos elaborados por los propios alumnos y profesores de cada centro, cuando están depositados en la biblioteca para ser utilizados por otros grupos.

Ahora bien, el plan lector y concretamente, las bibliotecas de los centros, no pueden conformarse con la disponibilidad y préstamo de medios, sino que deben desarrollar una tarea de orientación y animación sobre el uso de los mismos (animación lectora). A la función técnica de gestión de la biblioteca escolar (registro, clasificación, organización, mantenimiento, préstamo...), se debe sobreponer una función orientadora con un importante componente pedagógico respecto a profesores y alumnos, para informarles sobre qué medios utilizar y cómo hacerlo. Un paso más se da cuando se potencian en el centro las actividades de animación a la lectura, destinadas a motivar y desarrollar la habilidad lectora. (Calvo, 1999).

En todo caso, no podemos conformarnos con la disponibilidad de recursos didácticos. Es preciso analizar las actitudes y capacidades de profesores y alumnos para su uso. De poco serviría contar con material audiovisual o digitalizado de importante interés técnico y educativo, si no se utiliza por falta de interés o preparación en los profesores. Pueden existir valiosos trabajos realizados por grupos de alumnos y profesores que son desconocidos por otros compañeros. Puede ocurrir, en sentido contrario, que a partir de pocos medios o medios con pobre potencial educativo, la óptima utilización que haga de ellos el profesor, de lugar a resultados superiores a lo esperable.

En definitiva, la utilización de recursos didácticos en torno a los temas transversales nos remite al importante tema de la formación profesional inicial y permanente del profesorado y al establecimiento en los centros de condiciones de trabajo, como tiempo liberado de docencia, que hagan posible su utilización eficaz.

Existe la tendencia a identificar recursos con instrumentos, en torno a los cuales analizamos su disponibilidad y uso como se ha indicado respecto a los libros, los medios audiovisuales e informáticos. No obstante, desde un concepto ampliado de medios, al estudiar las características de la enseñanza emergente y concretamente, de la enseñanza de los temas transversales, se puede hacer referencia a las propias experiencias personales que sobre ellos han vivido o viven profesores y alumnos, experiencias de la más variada índole, que tienen lugar dentro o fuera del contexto escolar, que se desarrollan de manera espontánea o pueden ser provocadas. Se incluyen en este sentido, vivencias relativas a la superación de una enfermedad, al cuidado de un familiar, al cuidado de mascotas, vivencias relativas a fenómenos climáticos y ambientales, vivencias de voluntariado, etc. 
Esta utilización de las experiencias personales, que ha sido recomendado por la UNESCO (1986) como método emocional, especialmente apto para la educación en cuestiones sociales, implica la descripción y análisis de su desarrollo, así como de la actuación de sus protagonistas y la propuesta de alternativas.

A estas dos importantes fuentes de recursos (medios impresos, audiovisuales e informáticos y experiencias personales), hay que agregar otra más, consistente en la toma de contacto con la realidad natural y sociocultural (Austin, 2009). Una salida escolar cuidadosamente planificada para realizar observaciones y recoger datos sobre la naturaleza y la climatología, una visita a una granja escuela para obtener información sobre la vida de plantas y animales, sobre la elaboración de productos... Una salida para asistir a una representación teatral sobre un tema social actual...La realización de un "proyecto con alma” (Martín García, 2015) como podría ser la convivencia con personas mayores en una residencia de la tercera edad... Normalmente el entorno de los centros está lleno de posibilidades, a veces poco utilizadas.

Es evidente que el profesorado tiene en la actualidad grandes limitaciones en cuanto a tiempo y carga de trabajo para poder llevar a cabo algunas de estas actividades. Pero también lo es que en muchas ocasiones se desaprovechan oportunidades. Quizás, en este sentido, también podríamos considerar como un valioso recurso la colaboración de las asociaciones de madres y padres (AMPAS) de los colegios en la propuesta de actividades y en el apoyo en su realización.

\section{ESTRATEGIAS DE INNOVACIÓN}

La introducción de los temas transversales en el sistema educativo obedece en principio a un modelo innovador de carácter descendente. La administración educativa en España, ha seguido las orientaciones de organizaciones educativas internacionales desde mediados del siglo XX en el sentido de una actualización científica y social de los contenidos de enseñanza, con la introducción de temas y valores sociales (UNO, UNESCO, OEA, UE...).

De hecho, en las principales leyes educativas de nuestro país durante las últimas décadas, se van incluyendo temas transversales en sus finalidades, principios y objetivos generales. También en los decretos de currículo aparecen puntualmente dentro de algunos núcleos de contenido. Ahora bien, existe una notable variabilidad en las definiciones de estos temas. Se incide de manera intensa en algunos, mientras que otros casi no merecen atención.

De aquí la necesidad de que exista un cierto nivel de autonomía en la capacidad de decisión de los centros educativos y los profesores. Será, a partir del análisis de las características socioculturales, académicas y personales de sus alumnos, como se pueda elaborar una programación adaptada de la enseñanza de estos temas. Se podría afirmar que esta enseñanza debería ser evolutiva, adaptada y personalizada. (variaciones en la relevancia de los temas en función del paso del tiempo, de las características de la comunidad, de las características de cada alumno).

Esta programación y desarrollo adaptado de la enseñanza de los temas transversales a nivel de centro y de aula hace necesaria la puesta en práctica de procesos de investigación activa basados en la reflexión individual y grupal. A la reflexión del profesor sobre su actuación individual en 
el aula, se acompaña la reflexión grupal del equipo de profesores y especialistas en cada centro educativo. La realización de esta reflexión/investigación sobre la práctica se ha de fundamentar en el consenso de todos los profesionales participantes, como garantía para la obtención de información amplia y válida. Asimismo, ha de ser respetuosa con los derechos personales y profesionales de todos los miembros del grupo.

Los resultados de una investigación basada en la reflexión sobre la práctica deben constituir el punto de partida de iniciativas para la innovación de la enseñanza en las aulas y en los centros escolares. Con ello se potenciaría un modelo de innovación de carácter ascendente, complemento necesario del modelo administrativo descendente.

Existe otra forma de innovación en la enseñanza de los temas transversales, de carácter horizontal, cuando un centro toma contacto con otras instituciones o con otros centros. Se puede hacer referencia a las experiencias realizadas en zonas de población especialmente deprimida. El sistema educativo puede establecer vínculos de intervención coordinada con instituciones laborales, sanitarias o asistenciales.

Desde una perspectiva interna al sistema educativo, el establecimiento de redes de colaboración entre centros se manifiesta como una posibilidad de creciente interés pedagógico. En este sentido, se puede tomar como referencia el Proyecto de Escuelas Asociadas a la UNESCO, más de nueve mil colegios que en todo el mundo se esfuerzan por desarrollar aprendizajes relativos a la convivencia pacífica y la conservación del medio ambiente y del patrimonio natural y cultural. Así A. Tutor (2007), hace referencia a proyectos como los siguientes:

\section{a) El Proyecto del Mediterráneo occidental}

Creado por la UNESCO en 1992 para promover la realización de actividades educativas entre nueve países sobre el tema "aprender a vivir juntos en torno al Mediterráneo, educación en función del desarrollo sostenible" En este proyecto se trabajaron temas como el patrimonio cultural, la biodiversidad y los recursos naturales de la zona, el desarrollo sostenible y el agua, migraciones y sociedades multiculturales, diversidad lingüística y diálogo entre culturas y religiones. Los principales objetivos del proyecto fueron sensibilizar a los alumnos sobre estos temas y estimular su participación activa en los mismos. Como resultado de este proyecto, en 1994 surge El proyecto Atlántico, que tiene su campo de proyección en países con costas en este océano. Escuelas gallegas y portuguesas han participado activamente en el mismo.

b) El proyecto "Patrimonio: participación de los jóvenes en la conservación y promoción del patrimonio mundial"

En él participan escuelas de dieciséis países de todo el mundo y sus objetivos se proyectan en extender el conocimiento de la Convención del Patrimonio Mundial, en fomentar la conservación de los oficios tradicionales, establecer la cooperación entre los países participantes, dar a conocer las culturas y promover el diálogo.

c) El proyecto "Youth \& Change: Jóvenes para el cambio, hacia estilos de vida sostenibles"

Se trata de un proyecto que surge de la cooperación del Programa de las Naciones Unidas para el Medio Ambiente y la UNESCO. En él se incide en el desarrollo de actitudes y capacidades 
para construir un futuro sostenible. Se trata de un programa especialmente dirigido a jóvenes y abarca temas muy diversos como el consumo, la emisión de $\mathrm{CO} 2$, el ahorro y cambio energético...

La Red de Escuelas Asociadas a la UNESCO ha alcanzado una gran extensión y dinamismo y ello quizás se debe a factores como:

- La importancia vital de los temas en que se implica: convivencia, paz, medio ambiente, derechos humanos...

- La no exigencia de condiciones excepcionales para participar en sus proyectos: se respetan las características de los sistemas educativos y paralelamente al desarrollo de temas convencionales, se incide especialmente en los mencionados.

- La comunicación y colaboración entre los centros escolares que participan en sus proyectos, de tal modo que todos pueden beneficiarse de iniciativas y avances conseguidos en cada uno de ellos.

- La compatibilidad de las preocupaciones y necesidades locales con las de carácter universal.

No obstante, el modelo horizontal de innovación en la enseñanza de los temas transversales se manifiesta también en redes de menor amplitud, constituidas por colegios de una zona o distrito con necesidades y aspiraciones convergentes.

\section{CONCLUSIONES}

La organización de los contenidos de enseñanza según áreas o disciplinas convencionales, herencia de la organización científica del conocimiento, se manifiesta cada vez más insuficiente para responder a importantes necesidades educativas de los alumnos vinculadas a relevantes cuestiones sociales y académicas que rebasan el ámbito de cada asignatura. Por ello, se hace patente la necesidad de potenciar el trabajo interdisciplinar y en equipo de profesores y especialistas.

En la planificación administrativa de la enseñanza, según las últimas leyes fundamentales a nivel estatal y autonómico, se incrementa la presencia de los temas transversales, pero con cierta ambigüedad. No se parte de un análisis justificativo, ni se precisa por qué en unos temas se incide más intensamente y menos en otros. Se les confiere importancia a nivel de fines, principios, objetivos generales y competencias, pero su presencia en los núcleos de contenido es esporádica y puntual. En algún momento (LOE, 2006), se planificó una asignatura obligatoria (educación para la ciudadanía) sobre algunos de estos temas en las etapas de educación primaria y secundaria obligatoria, pero en estos momentos tan solo se contempla una asignatura optativa (educación en valores, LOMCE, 2013), con lo que el modelo curricular oficial sigue considerándolos como temas a impartir de forma implícita a través de las disciplinas convencionales.

La ambigüedad administrativa en relación con estos temas confiere de hecho un papel relevante a centros escolares y profesores, que serán los responsables de darles presencia real en las tareas cotidianas de enseñanza y aprendizaje. Los centros escolares, en su proyecto educativo, como manifestación de su autonomía pedagógica, pueden adaptar el tratamiento de estos contenidos a las 
características socioculturales y académicas de sus alumnos. Y los profesores, como profesionales capacitados para diagnosticar y reconducir los procesos de aprendizaje, van a ser los responsables de su desarrollo en las aulas.

Se consideran cualidades necesarias en el profesorado no solo el conocimiento de los temas, sino su capacidad de entrega motivada al trabajo en los mismos, como principio necesario para una transformación cualitativa (no sustitución), de los contenidos convencionales. Se considera asimismo, que la formación profesional docente debería potenciar tanto en su periodo inicial como permanente, capacidades relativas al trabajo en equipo con otros profesores, especialistas e instituciones, a la función tutorial proyectada en el grupo y en el alumno y las capacidades de observación y de reflexión sobre la práctica.

En el análisis de recursos para la enseñanza y aprendizaje de los temas transversales, es preciso hacer referencia a su variedad y disponibilidad, así como al uso que se hace de los mismos. La biblioteca escolar, dentro del plan lector, puede constituir un valioso centro de recursos (impresos, audiovisuales, informáticos...) para su mantenimiento y préstamo, para la orientación a profesores y alumnos en su uso, para la animación lectora.

Tan importante lo es el recurso de las experiencias que alumnos y profesores han vivido o pueden vivir en torno a los temas transversales. La descripción y análisis de las experiencias vividas, puede ampliarse con nuevas experiencias provocadas (salidas) o con el análisis de las que de manera espontánea se desarrollan en el contexto escolar (incidentes críticos).

Dentro del amplio, pero ambiguo marco de innovación descendente propuesto por la administración educativa, profesores y centros pueden protagonizar una innovación cualitativa, adaptada y eficaz en la enseñanza y aprendizaje de los temas transversales, en la medida en que pongan en práctica procesos de investigación basados en la reflexión individual y grupal sobre la práctica en las aulas y los centros. Esta innovación, de carácter ascendente, puede ampliarse en la medida en que se transforme en horizontal a través de la promoción de vínculos de coordinación y la realización de proyectos con otras instituciones y centros escolares. El Proyecto de Escuelas Asociadas a la UNESCO es representativo de las posibilidades de dicho modelo horizontal.

\section{REFERENCIAS BIBLIOGRÁFICAS}

Austin, R. (2009). Deja que el mundo exterior entre en el aula. Madrid: Morata.

Ávila, $\mathrm{M}^{\mathrm{a}}$ (2016). La educación para la ciudadanía en la formación inicial de maestros. Foro de Educación, 14(20) 153-175. DOI: https://doi.org/10.14516/fde.2016.014.020.009

Calvo, B. (1999). Animación a la lectura. Educación y biblioteca., 11(100), 5-7. Recuperado de https://gredos.usal.es/handle/10366/115396

Cavallo, G. y Chartier, R. (2001). Historia de la lectura. Madrid: Taurus.

Clemente, $M^{\mathrm{a}}$. (2004). Literatura y cultura escrita. Madrid: Morata.

Decreto 105/2014 de 4 de septiembre por el que se establece el Currículo de la Educación Primaria en la Comunidad Autónoma de Galicia. $D O G$ n. 171 de 9 de septiembre. 
LODE (1985). Ley Orgánica 8/1985 de 3 de julio reguladora del Derecho a la Educación. BOE n. 159 de 4 de julio.

LOE (2006). Ley Orgánica 2/2006 de 3 de mayo de Educación. BOE n. 106 de 4 de mayo.

LOGSE (1990). Ley Orgánica 1/1990 de 3 de octubre de Ordenación General del Sistema Educativo. BOE n. 238 de 4 de octubre.

LOMCE (2013). Ley Orgánica 8/2013 de 9 de diciembre para la mejora de la calidad de la enseñanza. $B O E$ n. 295 de 10 de diciembre.

Lluch, G. y Zayas, F. (2015). Leer en el centro escolar. Barcelona: Octaedro.

Martín García, X. (2015). Proyectos con alma. Trabajo por proyectos con servicio a la comunidad. Barcelona: Graó.

Orden ECI/3857/2007 de 27 de diciembre por la que se establecen los requisitos para la verificación de los títulos universitarios oficiales que habilitan para el ejercicio de la profesión de Maestro de Educación Primaria. BOE n. 312, de 29 de diciembre.

PISA (2018). Informe de evaluación. Recuperado de https://elpais.com/elpais/2016/12/05/media/1480958752 164797.html [Consultado el 1 de marzo de 2018].

Pujolás, J. (2008). 9 Ideas clave. El aprendizaje cooperativo. Barcelona: Graó.

Real Decreto 1393/2007 de 29 de octubre por el que se establece la ordenación de las enseñanzas universitarias oficiales. BOE n. 260 de 3 de octubre.

Real Decreto 1791/2010 de 30 de diciembre por el que se aprueba el Estatuto del Estudiante Universitario. BOE n. 318 de 31 de octubre.

Rosales, C. (2011). Tiempo escolar, tiempo de vida. Educación y futuro digital, 1, 46-59.

Rosales, C. (2015). Los temas transversales en el aula. Santiago: Andavira.

Rosales, C. (2017). Alumnos, maestros, colegios e incidentes. Santiago: Andavira.

Stenhouse, L. (1984). Investigación y desarrollo del currículum. Madrid: Morata.

Tutor, A. (2007). La Red de Escuelas Asociadas a la UNESCO en Aragón. Naturaleza aragonesa, 19, julio-diciembre, 72-77.

UNESCO (1986). Didáctica sobre cuestiones universales de hoy. Barcelona: Teide.

Zeichner, K. M. (2010). La formación del profesorado y la lucha por la justicia social. Madrid: Morata. 